\section{Uso de redes neurais artificiais para classificação de municípios quanto à vulnerabilidade social no Estado do Rio Grande do Norte, Brasil}

\section{The use of artificial neural networks to classify the social vulnerability of municipalities in Rio Grande do Norte State, Brazil}

\author{
Uso de redes neuronales artificiales para \\ la clasificación de municipios respecto a la \\ vulnerabilidad social en Estado del \\ Rio Grande do Norte, Brasil
}

Denise Guerra Wingerter 1,2 Emelynne Gabrielly de Oliveira Santos 2 Isabelle Ribeiro Barbosa 2

\section{Resumo}

O objetivo foi aplicar as redes neurais artificiais para classificar os municípios do Estado do Rio Grande do Norte, Brasil, de acordo com sua vulnerabilidade social. Estudo ecológico que utilizou 17 variáveis que refletissem os indicadores epidemiológicos, demográficos, socioeconômicos e educacionais para $o$ ano de 2010. As fontes pesquisadas foram o Atlas do Desenvolvimento Humano no Brasil e o Instituto Brasileiro de Geografia e Estatística. Para a classificação dos municípios, foram aplicadas as redes neurais artificiais, dos tipos PNN e Multilayer feedforward, resultando a classificação em cinco categorias de vulnerabilidade: muito alta, alta, média, baixa e muito baixa. A fase de treinamento das redes utilizou os valores de mínimo, máximo, percentis 25 e 75 e mediana das 17 variáveis selecionadas. A rede Multilayer feedforward com seis nós apresentou os melhores resultados. Os municípios da região metropolitana (Natal, Parnamirim), das microrregiões do Seridó oriental e ocidental (Caicó, Currais Novos, São José do Seridó, Jardim do Seridó, Parelhas, Carnaúba dos Dantas) apresentaram níveis mais baixos de vulnerabilidade. Os municípios de alta e muito alta vulnerabilidade encontramse na mesorregião do Leste potiguar: nas microrregiões do Litoral Nordeste (municípios de João Câmara, Touros, Caiçara do Rio dos Ventos) e do Litoral Sul (Nísia Floresta, São José do Mipibu, Arês, Canguaretama). A rede neural classificou os municípios com elevada precisão, destacando os que possuem extrema vulnerabilidade daqueles que detêm os melhores indicadores sociais.

Redes Neurais de Computadores; Vulnerabilidade Social; Indicadores Sociais

\author{
Correspondência \\ D. G. Wingerter \\ Secretaria Estadual de Saúde Pública do Rio Grande do Norte. \\ Av. Deodoro da Fonseca 730, 50 andar, sala 10, Natal, RN \\ 59025-600, Brasil. \\ denisegw@gmail.com \\ 1 Secretaria Estadual de Saúde Pública do Rio Grande do Norte, \\ Natal, Brasil. \\ 2 Universidade Federal do Rio Grande do Norte, Natal, Brasil.
}




\section{Introdução}

A compreensão das dinâmicas populacionais dentro dos territórios constitui elemento fundamental ao planejamento e organização dos serviços públicos, uma vez que o território consiste em espaço social físico e historicamente constituído, onde uma determinada população se organiza a partir de diferentes classes sociais, culturas, condições de vida e de acesso aos serviços públicos. Essas especificidades interferem ou atuam condicionando a vida das pessoas e consequentemente a atuação das políticas públicas 1 .

Um dos indicadores utilizados em políticas públicas para a avaliação da qualidade de vida de uma população é o índice de vulnerabilidade social, que se configura como uma dinâmica de interdependências em que indivíduos ou grupo desses fazem parte de um processo de exclusão social em que se entrelaçam diversos fatores; dente eles, destacam-se os fatores econômicos, sociais, demográficos, biológicos e existenciais. Uma situação de vulnerabilidade restringe as capacidades relacionais de afirmação no mundo, gerando fragilização do indivíduo ou da comunidade 2 .

Nesse contexto, a identificação de áreas de maior vulnerabilidade e as análises dentro de um território podem ser realizadas a partir da utilização de técnicas que permitam a obtenção de estimativas confiáveis que apresentem autonomia e precisão para filtrar, definir e classificar as variáveis mais representativas para a consolidação de um modelo de análise, como as redes neurais artificiais (RNA) 3 .

Desse modo, as RNA, configuradas como modelos computacionais baseados em modelagem matemática de problemas, inspirados pelo funcionamento do sistema nervoso animal (em particular o cérebro), são capazes de realizar o aprendizado de máquina e reconhecer padrões e foram desenvolvidas para realizar tarefas complexas que são difíceis de resolver utilizando programação baseada em regras comuns 4.

Logo, análises realizadas a partir das RNA podem contribuir para o reconhecimento das áreas de maior vulnerabilidade social e refletir, portanto, no estabelecimento de políticas sociais voltadas para o enfrentamento das desigualdades e iniquidades socioeconômicas em diversas regiões. Assim, o presente estudo visa à aplicação das RNA para classificar os municípios do Estado do Rio Grande do Norte, Brasil, de acordo com sua vulnerabilidade social.

\section{Métodos}

Trata-se de um estudo ecológico que utilizou informações agregadas para os 167 municípios do Estado do Rio Grande do Norte. A proposta desse estudo foi realizar a classificação dos municípios baseada nas necessidades da população, considerando variáveis que refletissem a vulnerabilidade social para o processo de classificação.

Foram selecionadas 17 variáveis que abrangeram questões demográficas, epidemiológicas, socioeconômicas e educacionais, a saber: (v1) taxa de mortalidade infantil, (v2) taxa de mortalidade até 5 anos de idade, (v3) índice de Gini, (v4) percentual da população em domicílios com densidade > 2, (v5) percentual de mulheres de 10 a 17 anos que tiveram filhos, (v6) percentual de mães chefes de família sem Ensino Fundamental e com filho menor, (v7) percentual de crianças de 6 a 14 anos fora da escola, (v8) percentual de pessoas em domicílios em que ninguém tem Ensino Fundamental completo, (v9) percentual de pessoas de 15 a 24 anos que não estudam, não trabalham e são vulneráveis, (v10) percentual de pessoas em domicílios vulneráveis à pobreza e em que ninguém tem Ensino Fundamental completo, (v11) percentual de vulneráveis e dependentes de idosos, (v12) percentual de pessoas em domicílios com abastecimento de água e esgotamento sanitário inadequados, (v13) razão de dependência, (v14) taxa de analfabetismo de pessoas de 15 anos ou mais, (v15) percentual de pobres, (v16) percentual de vulneráveis à pobreza, (v17) percentual de crianças extremamente pobres.

As variáveis epidemiológicas foram obtidas do Atlas do Desenvolvimento Humano no Brasil 2013 (Programa das Nações Unidas para o Desenvolvimento; Instituto de Pesquisa Econômica Aplicada; Fundação João Pinheiro. http://atlasbrasil.org.br/2013/pt/consulta/, acessado em Fev/2019), e a base cartográfica foi coletada do Instituto Brasileiro de Geografia e Estatística (Geociências. https:// www.ibge.gov.br/geociencias/downloads-geociencias.html, acessado em Fev/2019), ambas são bases de dados de domínio público. 
Os dados obtidos para os municípios foram organizados pelo software Microsoft Office Excel 2007 (https://products.office.com/). Realizou-se análise preliminar das variáveis e sua descrição por meio dos valores mínimo, máximo e mediana. Os graus de correlação entre as variáveis foram avaliados a partir da análise de correlação linear de Pearson. As 17 variáveis mantidas no modelo não apresentavam colinearidade (apresentaram $\mathrm{r}<0.8$ ) 5. Essa análise tem por objetivo avaliar estatisticamente quais as informações e variáveis relacionadas ao problema em estudo que são realmente importantes para alimentar a RNA, de modo que o modelo consiga captar a variância de cada variável e sua influência nos resultados da rede; havendo colinearidade, tornar-se-á muito difícil haver variação em uma sem que haja em outra.

O processo de classificação dos municípios foi realizado, seguindo as etapas: (a) foram criados cinco municípios fictícios para a classificação da vulnerabilidade, a partir dos valores máximos, mínimos, da mediana e dos percentis 25 e 75 de cada variável do estudo: um "município de muito alta vulnerabilidade", que apresentou os valores máximos de cada variável; um "município de alta vulnerabilidade", que apresentou os valores dos percentis 75 de cada variável; um "município de média vulnerabilidade", com valores da mediana de cada variável; um "município de baixa vulnerabilidade", com valores dos percentis 25 de cada variável; e um "município de muito baixa vulnerabilidade", com os valores mínimos de cada variável; (b) a partir da criação desses cinco municípios fictícios, foi obtida uma amostra aleatória de 120 municípios entre "muito baixa vulnerabilidade" e "muito alta vulnerabilidade"; (c) na etapa de treinamento, foi indicado ao software a utilização de 50\% dos municípios fictícios para treinar a da rede neural artificial, os quais foram selecionados aleatoriamente pelo software para a realização dessa etapa; a outra parte foi usada na etapa de teste do modelo; (d) os dados dos 167 municípios do rn foram usados na fase de previsão da rede.

Foi utilizada a rede $P N N$, que é uma rede neural probabilística, na qual cada caso de treinamento é representado por um elemento da rede (um nó). Uma previsão para um caso com valor dependente desconhecido é obtida por meio da interpolação dos casos de um treinamento, sendo que é atribuído mais pesos aos casos vizinhos. Durante o treinamento, são encontrados os parâmetros ótimos de interpolação. Aplicou-se também a rede Multilayer feedforward (MLFN), que é composta por uma camada de input de nós, uma ou duas camadas ocultas e uma camada de output. Foram testadas as redes com dois a seis nós. As RNA do tipo perceptrons de múltiplas camadas são muito utilizadas por apresentarem maior simplicidade e facilidade de implementação. O número de camadas e nodos utilizados nas redes foi determinado de forma empírica, baseando-se na complexidade do problema em estudo, número de exemplos de treinamento, quantidade de ruído presente nos exemplos e distribuição estatística dos dados de treinamento. Geralmente, são aplicados diferentes métodos de treinamento, diferentes números de camadas e nodos com o intuito de avaliar a combinação que melhor atende aos requisitos, começando com um número de nodos e ir eliminando os nodos que apresentarem as ligações sinápticas mais fracas (menores pesos) 6.

Os resultados para as melhores redes incluem os níveis de erro nas fases de treinamento e teste, para cada rede. Nas métricas utilizadas para avaliar a qualidade do teste e da predição, a melhor rede foi aquela que apresentou o menor erro e o maior nível de confiança na classificação. Esse procedimento classificou os 167 municípios do Estado do Rio Grande do Norte em uma das cinco classificações para a vulnerabilidade, além de indicar o impacto de cada variável no modelo de previsão. Após a classificação, os resultados da vulnerabilidade foram apresentados em um mapa coroléptico para as cinco categorias de vulnerabilidade.

Para a realização das análises das RNA, foi utilizado o software NeuralTools (Decision Tools Suite 7.5.2; https://www.palisade.com/neuraltools/default.asp). Para análise descritiva das variáveis, foi utilizado o software SPSS (https://www.ibm.com/), e, para a produção do mapa, foi utilizado o Terraview 4.2.2 (http://www.dpi.inpe.br/terraview).

Por se tratar de estudo realizado com dados secundários, o presente estudo é dispensado de avaliação por Comitê de Ética em Pesquisa, em conformidade com a Resolução no 466 do Conselho Nacional de Saúde (CNS), de 12 de dezembro de 2012. 


\section{Resultados}

Dentre as redes neurais testadas, a MLFN com seis nós apresentou os melhores resultados para o conjunto de dados, conforme pode ser observado na Tabela 1.

$\mathrm{Na}$ Tabela 2, são apresentados os resultados das médias e valores mínimo e máximo das 17 variáveis do estudo, com os respectivos municípios que apresentaram tais valores. Os municípios que apresentaram os piores indicadores foram João Dias, Galinhos e Montanhas; já entre os municípios com as melhores condições, encontram-se Natal, Parnamirim e Caicó.

As variáveis que apresentaram maior impacto no modelo preditivo foram "percentual da população em domicílios com densidade > 2" (12,3\%), "percentual de vulneráveis à pobreza" (11,05\%) e a “taxa de analfabetismo de pessoas de 15 anos ou mais” (10,39\%). Já as variáveis com menor impacto foram: "percentual de vulneráveis e dependentes de idosos" (2,4\%) e "percentual de mulheres de 10 a 17 anos que tiveram filhos” (1,5\%). O ranking dos valores do impacto de cada variável está apresentado na Tabela 3.

A análise estatística aplicada ao resultado do modelo de previsão possibilitou a distribuição dos municípios do Rio Grande do Norte em cinco categorias, classificados a seguir: dez municípios que apresentaram os melhores indicadores e foram classificados como de "muito baixa vulnerabilidade";

Tabela 1

Estatística descritiva das variáveis epidemiológicas e socioeconômicas indicadoras de vulnerabilidade social dos municípios do Rio Grande do Norte, Brasil, 2010

\begin{tabular}{|c|c|c|c|c|c|}
\hline Variável & Mediana & Valor mínimo & Município & Valor máximo & Município \\
\hline Taxa de mortalidade infantil & 23,60 & 13,40 & Caicó & 36,50 & Montanhas \\
\hline Taxa de mortalidade de menores de 5 anos de idade & 25,41 & 14,45 & Caicó & 39,23 & Montanhas \\
\hline Índice de Gini & 0,50 & 0,40 & $\begin{array}{l}\text { Timbaúba dos } \\
\text { Batistas }\end{array}$ & 0,68 & Augusto Severo \\
\hline $\begin{array}{l}\text { Percentual da população em domicílios com } \\
\text { densidade }>2\end{array}$ & 32,07 & 15,23 & Jardim do Seridó & 50,23 & Vila Flor \\
\hline $\begin{array}{l}\text { Percentual de mulheres de } 10 \text { a } 17 \text { anos que tiveram } \\
\text { filhos }\end{array}$ & 33,80 & 0,00 & Jardim do Seridó & 89,70 & Água Nova \\
\hline $\begin{array}{l}\text { Percentual de mães chefes de família sem Ensino } \\
\text { Fundamental e com filho menor }\end{array}$ & 20,70 & 5,40 & Cruzeta & 55,60 & $\begin{array}{l}\text { Presidente } \\
\text { Juscelino }\end{array}$ \\
\hline Percentual de crianças de 6 a 14 anos fora da escola & 2,27 & 0,36 & $\begin{array}{l}\text { Riacho de } \\
\text { Santana }\end{array}$ & 12,24 & João Dias \\
\hline $\begin{array}{l}\text { Percentual de pessoas em domicílios em que ninguém } \\
\text { tem Ensino Fundamental completo }\end{array}$ & 40,27 & 15,01 & Parnamirim & 54,28 & Galinhos \\
\hline $\begin{array}{l}\text { Percentual de pessoas de } 15 \text { a } 24 \text { anos que não } \\
\text { estudam, não trabalham e são vulneráveis }\end{array}$ & 23,83 & 10,14 & Natal & 39,05 & Pilões \\
\hline $\begin{array}{l}\text { Percentual de pessoas em domicílios vulneráveis à } \\
\text { pobreza e em que ninguém tem Ensino Fundamental } \\
\text { completo }\end{array}$ & 28,41 & 8,90 & Natal & 43,33 & Galinhos \\
\hline Percentual de vulneráveis e dependentes de idosos & 53,00 & 1,15 & Galinhos & 10,26 & Paraná \\
\hline $\begin{array}{l}\text { Percentual de pessoas em domicílios com } \\
\text { abastecimento de água e esgotamento sanitário } \\
\text { inadequados }\end{array}$ & 15,25 & 0,73 & Parnamirim & 56,20 & $\begin{array}{l}\text { Jardim de } \\
\text { Angicos }\end{array}$ \\
\hline Razão de dependência & 53,97 & 39,76 & Parnamirim & 67,13 & Parazinho \\
\hline Taxa de analfabetismo de pessoas de 15 anos ou mais & 26,98 & 7,96 & Parnamirim & 38,86 & João Dias \\
\hline Percentual de pobres & 36,84 & 10,50 & Natal & 56,29 & João Dias \\
\hline Percentual de vulneráveis à pobreza & 64,19 & 29,01 & Parnamirim & 79,73 & João Dias \\
\hline Percentual de crianças extremamente pobres & 25,28 & 4,74 & Parnamirim & 59,65 & João Dias \\
\hline
\end{tabular}


Tabela 2

Análise da performance de seis redes neurais artificiais (RNA) para o conjunto de 17 variáveis.

\begin{tabular}{lcc}
\hline RNA & Previsões inadequadas no treinamento (\%) & Previsões inadequadas no teste (\%) \\
\hline PNN & 1,72 & 0,00 \\
MLFN 2 nós & 31,03 & 40,67 \\
MLFN 3 nós & 13,79 & 23,72 \\
MLFN 4 nós & 1,72 & 0,00 \\
MLFN 5 nós & 1,72 & 0,00 \\
MLFN 6 nós & 1,69 & 0,00 \\
\hline
\end{tabular}

MLFN: Multilayer feedforward.

\section{Tabela 3}

Ranking do impacto das variáveis no modelo de rede neural artificial (RNA) mais eficiente na classificação dos municípios estudados. Rio Grande do Norte, Brasil, 2010.

\begin{tabular}{|c|c|}
\hline Variável & Impacto (\%) \\
\hline Percentual da população em domicílios com densidade $>2$ & 12,13 \\
\hline Percentual de vulneráveis à pobreza & 11,05 \\
\hline Taxa de analfabetismo de pessoas de 15 anos ou mais & 10,39 \\
\hline Taxa de mortalidade de menores de 5 anos de idade & 7,88 \\
\hline Índice de Gini & 7,79 \\
\hline Percentual de pobres & 6,51 \\
\hline Percentual de crianças de 6 a 14 anos fora da escola & 6,51 \\
\hline Percentual de pessoas em domicílios em que ninguém tem Ensino Fundamental completo & 6,47 \\
\hline Percentual de mães chefes de família sem Ensino Fundamental e com filho menor & 5,57 \\
\hline Percentual de crianças extremamente pobres & 5,28 \\
\hline Percentual de pessoas de 15 a 24 anos que não estudam, não trabalham e são vulneráveis & 5,02 \\
\hline Percentual de pessoas em domicílios com abastecimento de água e esgotamento sanitário inadequados & 3,04 \\
\hline Razão de dependência & 3,01 \\
\hline Percentual de pessoas em domicílios vulneráveis à pobreza e em que ninguém tem Ensino Fundamental completo & 2,72 \\
\hline Taxa de mortalidade infantil & 2,66 \\
\hline Percentual de vulneráveis e dependentes de idosos & 2,40 \\
\hline Percentual de mulheres de 10 a 17 anos que tiveram filhos & 1,50 \\
\hline
\end{tabular}

44 foram considerados de "baixa vulnerabilidade"; 68 municípios com vulnerabilidades medianas foram classificados como de "média vulnerabilidade"; 55 municípios foram classificados com as piores condições de saúde e de contexto socioeconômico, sendo que, desses, 26 municípios foram classificados como de "alta vulnerabilidade" e 29 de "muito alta vulnerabilidade".

A Figura 1 traz a distribuição geográfica das categorias de vulnerabilidade, em que se destacam os municípios da região metropolitana (Natal, Parnamirim), das microrregiões do Seridó oriental e ocidental (Caicó, Currais Novos, São José do Seridó, Jardim do Seridó, Parelhas, Carnaúba dos Dantas) e da microrregião de Mossoró (Mossoró, Assú e Areia Branca) que apresentaram os melhores resultados das variáveis que representaram as condições socioeconômicas e de saúde. Os municípios de alta e muito alta vulnerabilidade encontram-se na mesorregião do Leste potiguar: nas microrregiões do Litoral Nordeste (municípios de João Câmara, Touros, Caiçara do Rio dos Ventos) e do Litoral Sul (Nísia Floresta, São José do Mipibu, Arês, Canguaretama). 

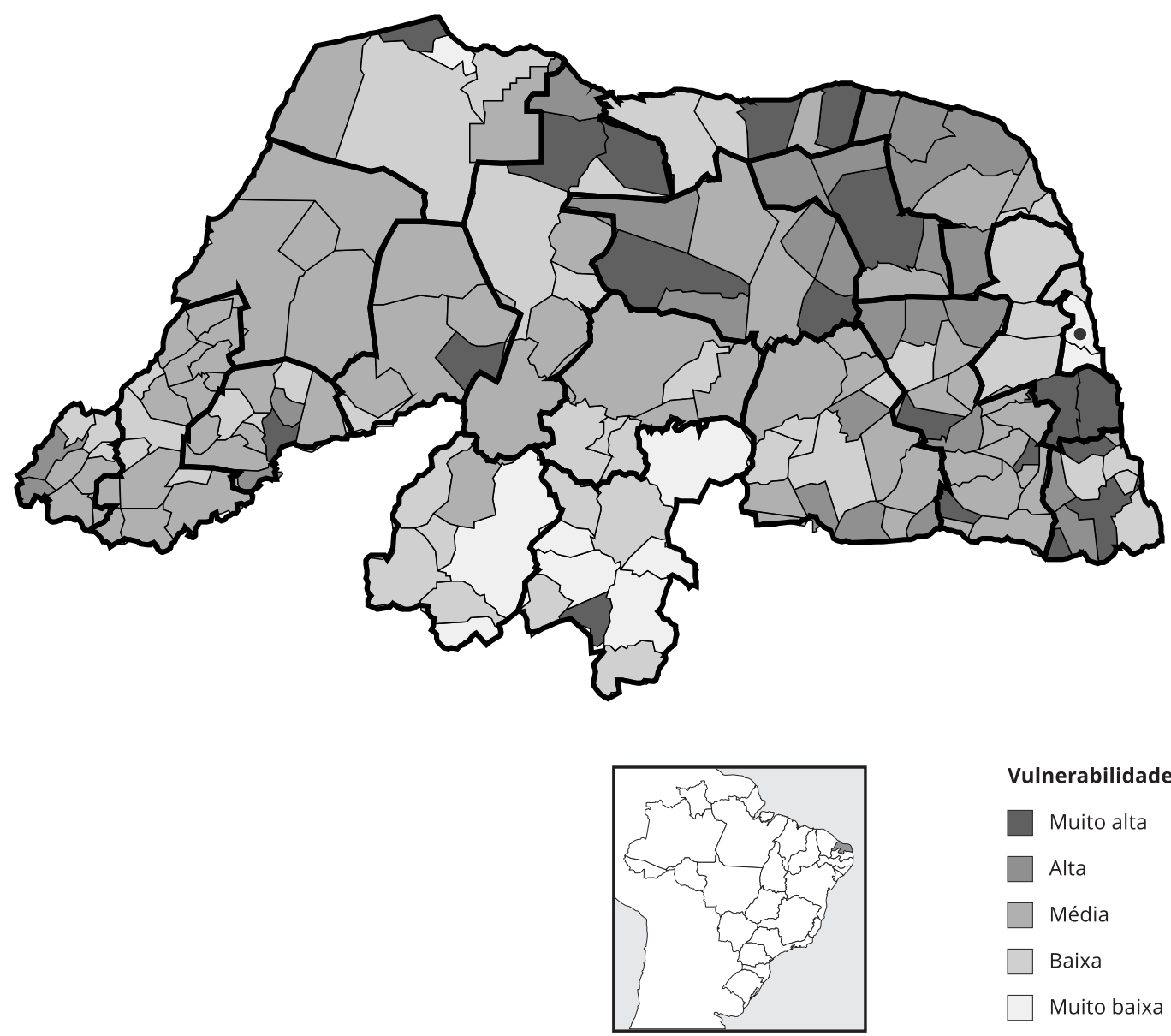

Vulnerabilidade

Muito alta

Alta

Média

Baixa

Muito baixa

\section{Discussão}

Os indicadores sintéticos, criados ou calculados a partir de outros indicadores para monitorar e avaliar áreas de vulnerabilidade social e também para alocação de recursos em saúde, são ferramentas úteis para a gestão pública, conforme Jannuzzi 7. Entretanto a dificuldade em classificar as áreas de maior vulnerabilidade social e que reflitam nas políticas sociais e de saúde enfatiza a necessidade de aprofundar a discussão e a análise de estratégias de fortalecimento de populações em situação de risco e dos recursos subjetivos para o enfrentamento dessa problemática 8 .

Para tanto, ao se pensar em estratégias que permitam reconhecer as áreas de maior vulnerabilidade social, é necessário agregar, ao critério geográfico, fatores que perpassem as dimensões sociais e, sobretudo, econômicas. Sendo assim, a escolha de variáveis que traduzam essas dimensões acaba se constituindo como um elemento fundamental para detectar tais áreas 9 .

Para Rosas et al. 8, algumas características devem ser consideradas na seleção das variáveis para compor as necessidades em saúde da população, como a disponibilidade de dados confiáveis. Para balizar esse estudo, utilizou-se variáveis que abrangeram aspectos demográficos, epidemiológicos, socioeconômicos e educacionais, provenientes de fontes de dados consolidadas. 
As variáveis relacionadas aos aspectos habitacionais, renda e educacionais chamaram atenção no estabelecimento do modelo de classificação dos municípios norte-rio-grandenses com maior vulnerabilidade social, como o percentual da população em domicílios com densidade $>2$, o percentual de vulneráveis à pobreza e a taxa de analfabetismo entre pessoas de 15 anos ou mais (Tabela 3). Esses dados, por sua vez, impactam diretamente nas necessidades básicas da população.

De acordo com o índice de desenvolvimento humano municipal (IDH-M), o Estado do Rio Grande do Norte ocupa a 16a posição em relação aos outros estados brasileiros, apresentando um índice de 0,684 , considerado como de médio desenvolvimento humano, sendo que $42 \%$ dos municípios apresentam IDH baixo; 56\%, médio; e somente 2\% apresentam IDHM considerado alto (Programa das Nações Unidas para o Desenvolvimento; Instituto de Pesquisa Econômica Aplicada; Fundação João Pinheiro. Atlas do desenvolvimento humano no Brasil 2013 http://atlasbrasil.org.br/2013/pt/ consulta/, acessado em Fev/2019).

Conforme as análises deste estudo, o Rio Grande do Norte possui 31\% dos seus municípios nas classificações de "muito baixa vulnerabilidade" e "baixa vulnerabilidade", sendo $6 \%$ de baixa vulnerabilidade e $25 \%$ de baixa vulnerabilidade, e outros $31 \%$ como alta vulnerabilidade (15\%) e muito alta vulnerabilidade (16\%), sendo que a maioria (38\%) encontra-se como média vulnerabilidade, panorama muito similar da descrição do IDH-M, denotando que o Rio Grande do Norte ainda permanece como lócus de desigualdade social 10, possuindo mais da metade dos seus municípios pertencentes à classificação média, alta ou muito alta de vulnerabilidade social.

Os municípios de Parnamirim e João Dias destacaram-se quanto aos valores mínimo e máximo das variáveis (Tabela 2), em que o primeiro, pertencente à região metropolitana, por apresentar os melhores resultados para seis das 17 variáveis, e o segundo, situado na região Oeste potiguar, na fronteira com o Estado da Paraíba, apresentando os piores valores para quatro variáveis, sendo três delas relacionadas à pobreza e uma à educação. Os municípios de muito pequeno porte, como João Dias, que contava, em 2010, com pouco mais de dois mil habitantes, são comumente preteridos dentro das políticas públicas dos estados, sendo essas mais focadas nos grandes centros urbanos 11, o que pode explicar o resultado da elevada vulnerabilidade.

A concentração de municípios com alta e muito alta vulnerabilidade na mesorregião do Leste potiguar observada na Figura 1 aponta para a necessidade de políticas públicas mais eficazes, monitoramento e avaliação nessas localidades, que concentram a maior parte dos municípios mais vulneráveis.

Por outro lado, as análises revelaram valores que traduzem positivamente a situação do Rio Grande do Norte quanto ao percentual de mulheres de 10 a 17 anos que tiveram filhos, o percentual de vulneráveis e dependentes de idosos e a taxa de mortalidade infantil. Assim, é possível refletir sobre as interseções entre as políticas públicas e o desenvolvimento de ações voltadas para prevenção da gravidez na adolescência no estado, assim como também é possível a avaliação para a concentração de municípios com baixa ou muito baixa vulnerabilidade na Mesorregião do Seridó, que indica que aquela região possui melhores indicadores, e que os resultados podem ser estendidos ao restante do estado.

\section{Considerações finais}

Desse modo, a aplicação das RNA para classificar os municípios do Estado do Rio Grande do Norte, de acordo com sua vulnerabilidade social, permitiu resultados com elevada precisão, destacando os que possuem extrema vulnerabilidade daqueles que detêm os melhores indicadores sociais. Este estudo pode servir de referencial para outras pesquisas comparativas, não só no âmbito estadual, mas alocadas a outras realidades, como também para a análise da situação de vulnerabilidade do estado para fins de alocação de políticas públicas georreferenciadas e que possam melhorar a qualidade de vida da população do estado objeto do estudo. 


\section{Colaboradores}

D. G. Wingerter colaborou na análise dos dados e redação do artigo. E. G. O. Santos e I. R. Barbosa participaram da coleta e análise de dados e redção do artigo.

\section{Informações adicionais}

ORCID: Denise Guerra Wingerter (0000-00030250-0255); Emelynne Gabrielly de Oliveira Santos (0000-0002-3890-4879); Isabelle Ribeiro Barbosa (0000-0002-1385-2849).

\section{Referências}

1. Duarte LS, Pessoto UC, Guimarães RB, Heimann LS, Carvalheiro JR, Cortizo CT, et al. Regionalização da saúde no Brasil: uma perspectiva de análise. Saúde Soc 2015; 24:472-85.

2. Oviedo RAM, Czeresnia D. O conceito de vulnerabilidade e seu caráter biossocial. Interface Comun Saúde Educ 2015; 19:237-50.

3. Rauber TW. Redes neurais artificiais. Vitória: Departamento de Informática, Universidade Federal do Espírito Santo; 2005.

4. Kovács ZL. Redes neurais artificiais. São Paulo: Editora Livraria da Física; 2002.

5. Maia AG. Econometria: conceitos e aplicações. São Paulo: Saint Paul Editora; 2017.

6. Fiorin DV, Martins FR, Schuch NJ, Pereira EB. Aplicações de redes neurais e previsões de disponibilidade de recursos energéticos solares. Revista Brasileira de Ensino de Física 2011; 33:1309.

7. Jannuzzi PM. Indicadores para diagnóstico, monitoramento e avaliação de programas sociais no Brasil. Revista do Serviço Público 2014; 56:137-60.

8. Rosas MA, Bezerra AFB, Duarte-Neto PJ. Uso das redes neurais artificiais na aplicação de metodologia para alocação de recursos da saúde. Rev Saúde Pública 2013; 47:128-36.

9. Costa MA, Marguti BO. Atlas da vulnerabilidade social nas regiões metropolitanas brasileiras. Brasília: Instituto de Pesquisa Econômica Aplicada; 2015.

10. Banco do Nordeste do Brasil. Necessidades de investimentos e convergência do PIB per capita. Brasília: Banco do Nordeste do Brasil; 2010.

11. Figueiredo VDM. Pequenos municípios e pequenas cidades do Estado do Rio Grande do Sul: contrastes, perfil do desenvolvimento e de qualidade de vida, 1980-2000 [Tese de Doutorado]. São Paulo: Instituto de Geociências e Ciências Exatas, Universidade Estadual Paulista; 2008. 


\section{Abstract}

The objective was to apply artificial neural networks to classify municipalities (counties) in Rio Grande do Norte State, Brazil, according to their social vulnerability. This was an ecological study using 17 variables that reflected epidemiological, demographic, socioeconomic, and educational indicators for the year 2010. The sources were the Human Development Atlas for Brazil and the Brazilian Institute of Geography and Statistics. For classification of the municipalities, the study applied the artificial neural networks of the PNN and Multilayer feedforward types, resulting in a classification in five categories of vulnerability: very high, high, medium, low, and very low. The networks' training phase used the minimum and maximum values, 25th and 75 th percentiles, and medians for the 17 selected variables. The Multilayer feedforward network with six nodes showed the best results. The municipalities from the Metropolitan Area (Natal, Parnamirim) and the eastern and western Seridó micro-regions (Caicó, Currais Novos, São José do Seridó, Jardim do Seridó, Parelhas, Carnaúba dos Dantas) showed the lowest levels of vulnerability. The municipalities with high and very high vulnerability were located in the East of the state, in the micro-regions of the Northeast Coast (João Câmara, Touros, Caiçara do Rio dos Ventos) and Southern Coast (Nísia Floresta, São José do Mipibu, Arês, Canguaretama). The neural network classified the municipalities with high precision, distinguishing those with extreme vulnerability from those with better social indicators.

Computer Neural Networks; Social Vulnerability; Social Indicators

\section{Resumen}

El objetivo fue aplicar las redes neuronales artificiales para clasificar los municipios del estado de Rio Grande do Norte, Brasil, de acuerdo con su vulnerabilidad social. Se realizó un estudio ecológico que utilizó 17 variables que reflejaron los indicadores epidemiológicos, demográficos, socioeconómicos y educacionales durante el año 2010. Las fuentes investigadas fueron: el Atlas de Desarrollo Humano en Brasil y el Instituto Brasileño de Geografía y Estadística. Para la clasificación de los municipios, se aplicaron las redes neuronales artificiales de los tipos PNN y Multilayer feedforward, resultando la clasificación en cinco categorías de vulnerabilidad: muy alta, alta, media, baja y muy baja. La fase de entrenamiento de las redes utilizó los valores: mínimo, máximo, percentiles 25 y 75 y mediana de las 17 variables seleccionadas. La red Multilayer feedforward con seis nudos presentó los mejores resultados. Los municipios de la región metropolitana ( $\mathrm{Na}$ tal, Parnamirim), de las microrregiones del Seridó oriental y ocidental (Caicó, Currais Novos, São José do Seridó, Jardim do Seridó, Parelhas, Carnaúba dos Dantas) presentaron niveles más bajos de vulnerabilidad. Los municipios de alta y muy alta vulnerabilidad se encuentran en la mesorregión del este potiguar: en las microrregiones del litoral nordeste (municipios de João Câmara, Touros, Caiçara do Rio dos Ventos) y del litoral sur (Nísia Floresta, São José do Mipibu, Arês, Canguaretama). La red neuronal clasificó los municipios con elevada precisión, destacando los que poseen extrema vulnerabilidad de aquellos que ostentan los mejores indicadores sociales.

Redes Neurales de la Computación; Vulnerabilidad Social; Indicadores Sociales
Recebido em 26/Fev/2019

Versão final reapresentada em 30/Out/2019

Aprovado em 04/Fev/2020 